\title{
On the rationality of the moduli space of Lüroth quartics
}

\author{
Christian Böhning • Hans-Christian Graf von Bothmer
}

Received: 26 May 2010 / Revised: 16 August 2011 / Published online: 31 August 2011

(C) The Author(s) 2011. This article is published with open access at Springerlink.com

\begin{abstract}
We prove that the moduli space $\mathfrak{M}_{L}$ of Lüroth quartics in $\mathbb{P}^{2}$, i.e. the space of quartics which can be circumscribed around a complete pentagon of lines modulo the action of $\mathrm{PGL}_{3}(\mathbb{C})$ is rational, as is the related moduli space of Bateman seven-tuples of points in $\mathbb{P}^{2}$.
\end{abstract}

\section{Introduction}

The 13-dimensional hypersurface in the parameter space $\mathbb{P}^{14}$ of plane quartics consisting of the closure of the locus of quartics which have an inscribed complete pentagon of lines, the Lüroth quartics, is classically called the Lüroth hypersurface and known to be irreducible, $\mathrm{SL}_{3}(\mathbb{C})$-invariant, and of degree 54 . In this paper we prove the rationality of the moduli space of Lüroth quartics, i.e. of the corresponding $\mathrm{SL}_{3}(\mathbb{C})$-quotient of the Lüroth hypersurface. Morley [12] described Lüroth quartics as branch curves of degree 2 rational maps of $\mathbb{P}^{2}$ defined by Geiser involutions. These involutions are determined by a net of cubics in $\mathbb{P}^{2}$ through seven distinct points in $\mathbb{P}^{2}$ no six of which lie on a conic. Thus we get a map from such seven-tuples of points to quartics, and certain configurations of points, called Bateman configurations, lead to Lüroth quartics. The original paper is [1]. Morley proves that there are generically 8 Bateman configurations leading to a Lüroth quartic. It is shown below that also the moduli space of Bateman seven-tuples of points is rational.

We would like to thank Edoardo Sernesi for bringing these interesting geometric questions to our attention and for helpful comments on the paper.

C. Böhning $(\bowtie) \cdot$ H.-C. G. von Bothmer

Göttingen, Germany

e-mail: boehning@uni-math.gwdg.de 


\section{Lüroth quartics and Bateman points}

Definition 2.1 A quartic curve $L \subset \mathbb{P}^{2}$ is called a Lüroth quartic if there exist $l_{1}, \ldots, l_{5} \in\left(\mathbb{C}^{3}\right)^{\vee}$ such that $\cup_{i \neq j}\left(\left(l_{i}=0\right) \cap\left(l_{j}=0\right)\right)$ consists of 10 points contained in $L$. We denote by $\mathfrak{P}_{L}$ the subset inside the parameter space $\mathbb{P}^{14}$ of plane quartic curves corresponding to Lüroth quartics.

A quartic curve $C$ is called a Clebsch quartic if there exist $l_{1}, \ldots, l_{5} \in\left(\mathbb{C}^{3}\right)^{\vee}$ such that $C=\left\{l_{1}^{4}+\cdots+l_{5}^{4}=0\right\}$. As in the previous item we denote by $\mathfrak{P}_{C}$ the subset of Clebsch quartics inside the space $\mathbb{P}^{14}$ of all plane quartics.

There is an $\mathrm{SL}_{3}(\mathbb{C})$-equivariant rational map $S_{4}: \mathfrak{P}_{C} \rightarrow \mathfrak{P}_{L}$ called the Scorza map which may be written symbolically as

$$
S_{4}=(\alpha \beta \gamma)(\alpha \beta \delta)(\alpha \gamma \delta)(\beta \gamma \delta) \alpha_{x} \beta_{x} \gamma_{x} \delta_{x}
$$

We refer to [4] for more explanation of the symbolical notation involved in this definition and to [5] for more information on the Scorza map itself. The book [8] is a historical account of invariant theory which also contains a readable account of the symbolic method. A modern treatment of the symbolic method can be found in [7]. It is known (cf. [5]) that $S_{4}$ induces a generically $1: 1$ map

$$
S_{4}: \mathfrak{P}_{C} \rightarrow \mathfrak{P}_{L}
$$

so that $\mathfrak{M}_{L}=\mathfrak{P}_{L} / \mathrm{SL}_{3}(\mathbb{C})$ and $\mathfrak{M}_{C}=\mathfrak{P}_{C} / \mathrm{SL}_{3}(\mathbb{C})$ are birational.

Theorem 2.2 The space $\mathfrak{M}_{C}$, hence also $\mathfrak{M}_{L}$, is rational.

Proof $\mathrm{By}[5], \mathfrak{P}_{C}$ is an open subset of the locus $\mathcal{K} \subset \mathbb{P}^{14}=\mathbb{P}\left(\operatorname{Sym}^{4}\left(\mathbb{C}^{3}\right)^{\vee}\right)$ given by the vanishing of the catalecticant invariant of quartics which is a degree 6 polynomial in the coefficients $A_{\underline{i}}$ of the quartic and defined as follows: for a quartic $C$ it is the determinant of the linear map

$$
l_{C}: \operatorname{Sym}^{2}\left(\mathbb{C}^{3}\right) \rightarrow \operatorname{Sym}^{2}\left(\mathbb{C}^{3}\right)^{\vee}
$$

given by acting on $C=\left(a x_{0}^{4}+b x_{0}^{3} x_{1}+\ldots\right)$ with a dual conic $Q \in \operatorname{Sym}^{2}\left(\mathbb{C}^{3}\right)$, $Q=A \frac{\partial^{2}}{\partial x_{0}^{2}}+B \frac{\partial^{2}}{\partial x_{0} x_{1}}+\ldots$, where we use dual bases in $\operatorname{Sym}^{2}(\mathbb{C})^{3}$ and $\operatorname{Sym}^{2}\left(\left(\mathbb{C}^{3}\right)^{\vee}\right) \simeq$ $\left(\operatorname{Sym}^{2}\left(\mathbb{C}^{3}\right)\right)^{\vee}$. We may view $l_{C}$ as induced by the multiplication map

$$
m: \operatorname{Sym}^{2}\left(\mathbb{C}^{3}\right) \times \operatorname{Sym}^{2}\left(\mathbb{C}^{3}\right) \rightarrow \operatorname{Sym}^{4}\left(\mathbb{C}^{3}\right) .
$$

Thus in summary $\mathfrak{M}_{L}$ is birational to $\mathcal{K} / \mathrm{SL}_{3}(\mathbb{C})$. By its definition, $\mathcal{K}$ fibres in an $\mathrm{SL}_{3}(\mathbb{C})$-equivariant way:

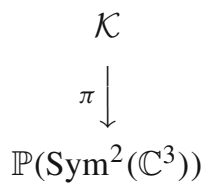


associating to a generic $C$ the kernel $\operatorname{ker}\left(l_{C}\right)$. The space $\mathbb{P}\left(\operatorname{Sym}^{2}\left(\mathbb{C}^{3}\right)\right)$ has a $\left(\mathrm{PSL}_{3}(\mathbb{C}), \mathrm{SO}_{3}(\mathbb{C})\right)$-section, namely a point. Hence $\mathcal{K} / \mathrm{SL}_{3}(\mathbb{C})$ is birational to $F / \mathrm{SO}_{3}(\mathbb{C})$ where $F$ is the generic fibre of $\pi$. In view of the isomorphism $\mathrm{SO}_{3}(\mathbb{C}) \simeq$ $\mathrm{PSL}_{2}(\mathbb{C})$, it remains to see that

$$
F \simeq \mathbb{P}\left(\operatorname{Sym}^{4}\left(\mathbb{C}^{3}\right) / \operatorname{Sym}^{2}\left(\mathbb{C}^{3}\right)\right) \simeq \mathbb{P}(V(8))
$$

as $\mathrm{PSL}_{2}(\mathbb{C})$-space where $V(n)$ is the representation of this group on the space of binary forms of degree $n$ for $n \in \mathbb{N}$. Note $\operatorname{Sym}^{4}\left(\mathbb{C}^{3}\right) \simeq V(8) \oplus V(4) \oplus V(0), \operatorname{Sym}^{2}\left(\mathbb{C}^{3}\right)=$ $V(4) \oplus V(0)$.

For once (1) is established we get the rationality of $\mathfrak{M}_{L}$ from that of $\mathbb{P}(V(8)) / \mathrm{PSL}_{2}$ $(\mathbb{C})$ (for a proof of the latter see $[2,9,10]$ ).

To see that (1) holds note that the fibre over $[Q]$ of $\pi$ consists of those quartics $C$ such that $Q$ is in the kernel of $l_{C}$, or equivalently of those linear forms on $\operatorname{Sym}^{4}\left(\mathbb{C}^{3}\right)$ which vanish on the image of $\operatorname{Sym}^{2}\left(\mathbb{C}^{3}\right)$ inside $\operatorname{Sym}^{4}\left(\mathbb{C}^{3}\right)$ under the map $m(Q, \cdot)$. Hence the claim.

We turn to the proof of rationality for the moduli space of Bateman 7-tuples of points, see [13] for background information.

Consider $2 \times 3$-matrices

$$
\left(\begin{array}{lll}
l_{1} & l_{2} & l_{3} \\
q_{1} & q_{2} & q_{3}
\end{array}\right)
$$

where the $l_{i}$ are linear, and the $q_{i}$ quadratic forms on $\mathbb{P}^{2}$, and remark that seven general points in $\mathbb{P}^{2}$ define a matrix of type (2) uniquely as syzygy matrix, and conversely a general matrix of type (2) determines seven points in $\mathbb{P}^{2}$ as its rank 1 locus. This can be made precise by defining $\mathbb{M}$ as the linear space of all matrices of type (2) which is acted on by the group

$$
\mathcal{G}=(\mathrm{GL}(V) \times \mathrm{GL}(W)) \ltimes G
$$

where $\mathbb{P}^{2}=\mathbb{P}(V)$ and $\operatorname{GL}(V)$ corresponds to coordinate changes, $W \simeq \mathbb{C}^{3}$ is the space of columns so that $\mathrm{GL}(W)$ corresponds to column operations, and $G$ is the group of row operations (adding a linear form times the first row to the second row). Then $\mathbb{M} / \mathcal{G}$ is a birational model for the moduli space of seven-tuples of points in $\mathbb{P}^{2}$.

Now seven general points in $\mathbb{P}^{2}$ can also be obtained as zero locus of a general global section in $\mathcal{T}_{\mathbb{P}^{2}}(1), \mathcal{T}_{\mathbb{P}^{2}}$ the tangent bundle on $\mathbb{P}^{2}$, and by Bott's theorem $H^{0}\left(\mathcal{T}_{\mathbb{P} 2}(1)\right)=$ $V(1,2)$, denoting by $V(a, b)$ the irreducible representation of $\operatorname{SL}(V)$ whose highest weight has numerical labels $a, b$ (so e.g. $\left.V(1,0)=V, V(0,1)=V^{*}\right)$. Thus we may also view $V(1,2)$ as a parameter space of seven points in $\mathbb{P}^{2}$ and we can relate this to the preceding matrix picture as follows: the $\mathcal{G}$-representation corresponding to the space of matrices of type (2) is

$$
V^{*} \otimes W+\mathrm{Sym}^{2} V^{*} \otimes W
$$


Acting by an element of $\mathcal{G}$ we may achieve that a general matrix of type (2) can be put in the form

$$
\left(\begin{array}{ccc}
x & y & z \\
q_{1} & q_{2} & q_{3}
\end{array}\right) .
$$

The stabilizer of matrices of this form inside $\mathcal{G}$ is isomorphic to $\operatorname{GL}(V) \ltimes G$. This means that we now have an isomorphism $\mathrm{GL}(V) \simeq \mathrm{GL}(W)$ and as $\operatorname{SL}(V)$-representation the full space of matrices decomposes

$$
V^{*} \otimes V+\operatorname{Sym}^{2} V^{*} \otimes V=(V(0,0)+V(1,1))+(V(1,2)+V(0,1))
$$

where $V(0,0)$ corresponds to our choice of $(x, y, z)$ in the first row. Acting with an element of the group of row operations $G$, we see that the $\mathcal{G}$-action on the space of all matrices of type (2) has a $\mathrm{GL}(V)$-section which as $\operatorname{SL}(V)$-representation is $V(1,2)$. Thus we see the birational isomorphism between $\mathbb{M} / \mathcal{G}$ and $\mathbb{P}(V(1,2)) / \operatorname{SL}(V)$ explicitly. This description is due to Katsylo and can be found in [11].

In other words, after the choice of the section (3), the space of matrices decomposes as in (4), and the points a matrix defines may be seen as the $V(1,2)$-component with respect to this decomposition.

Definition 2.3 An unordered collection of seven points $P_{1}, \ldots, P_{7}$ in $\mathbb{P}^{2}$ is said to be a Bateman seven-tuple of points in $\mathbb{P}^{2}$ if it is defined by a matrix of type (2) where the $l_{i}$ are the partial derivatives of a quadratic form $Q$ on $\mathbb{P}^{2}$ and the $q_{i}$ the partial derivatives of a cubic form $C$ on $\mathbb{P}^{2}$. We denote their moduli space for projective equivalence by $\mathfrak{M}_{B}$.

Remark 2.4 Morley [12] showed that $\mathfrak{M}_{B}$ is an eight to one cover of $\mathfrak{M}_{L}$ via taking the branch curve of a degree 2 map of $\mathbb{P}^{2}$ defined by a Geiser involution associated to the Bateman points (see also [13]).

What is the representation-theoretic meaning of the concept of Bateman points?

Lemma 2.5 There is a unique bilinear map

$$
b: V(2,0) \otimes V(0,3) \rightarrow V(1,2)
$$

and the image of the pure tensors in $V(2,0) \otimes V(0,3)$ under $b$ may be identified with the Bateman seven-tuples of points. More precisely, let the Bateman seven-tuple be defined by $Q$ and $C$ as in Definition 2.3. Let $Q^{*} \in V(2,0)$ be the quadric dual to $Q$ (recall that there is a degree 2 equivariant map $V(0,2) \rightarrow V(2,0)$ which corresponds to taking the adjugate of a symmetric matrix). Then $b\left(Q^{*}, C\right) \in V(1,2)$ is the associated Bateman seven tuple, using the correspondence between matrices and point sets encoded in the decomposition (4).

Proof Let us start with $Q$ and $C$ and consider the unique equivariant maps (derivatives)

$$
\begin{aligned}
& d_{2}: V(2,0) \otimes V(0,2) \rightarrow V(1,1)+V(0,0), \\
& d_{3}: V(2,0) \otimes V(0,3) \rightarrow V(1,2)+V(0,1) .
\end{aligned}
$$


In coordinate free terms, $d_{2}$ and $d_{3}$ are contractions with the identity. Then the matrix of the partials of $Q$ and $C$ is, in the identification made in decomposition (4), nothing but the pair

$$
\left(d_{2}\left(Q^{*}, Q\right), d_{3}\left(Q^{*}, C\right)\right)
$$

In fact one calculates that $d_{2}\left(Q^{*}, Q\right) \in V(0,0)$ (it suffices to check this for a particular quadric by equivariance). Note that

$$
\begin{aligned}
& d_{2}\left(Q^{*}, Q\right)=\sum_{i=1}^{3} \frac{\partial}{\partial e_{i}}\left(Q^{*}\right) \otimes \frac{\partial}{\partial x_{i}}(Q), \\
& d_{3}\left(Q^{*}, C\right)=\sum_{i=1}^{3} \frac{\partial}{\partial e_{i}}\left(Q^{*}\right) \otimes \frac{\partial}{\partial x_{i}}(C)
\end{aligned}
$$

where $e_{i}$ and $x_{j}$ are dual coordinates in $\mathbb{C}^{3}$ and $\left(\mathbb{C}^{3}\right)^{*}$. So the whole point of taking derivatives with respect to the quadric $Q^{*}$ dual to $Q$ is to obtain a matrix in normal form to achieve consistency with the choice of section made there. This proves the Lemma.

Now consider the isomorphism

$$
m: \Lambda^{2} V(2,0) \rightarrow V(2,1)
$$

This may be interpreted as a natural skew symmetric matrix $M$ on the space $V(2,0)$ with entries linear forms on the space $V(1,2)$ of points. Now the map $V(2,0) \otimes$ $V(2,0) \rightarrow V(2,1)$ given by $m$ is just the basic map $\vartheta$ on page 252 of [3]. For the definition of $\vartheta$, note that quite generally, abbreviating $S^{m}:=\operatorname{Sym}^{m} \mathbb{C}^{3}$ and $D^{n}=$ $\operatorname{Sym}^{n}\left(\mathbb{C}^{3}\right)^{\vee}$, we have a map

$$
\Theta:\left(S^{a} \otimes S^{b}\right) \otimes D^{c} \rightarrow\left(S^{a-1} \otimes S^{b-1}\right) \otimes D^{c+1}
$$

given by

$$
\Theta=\sum_{\sigma \in \mathfrak{S}_{3}}(-1)^{\operatorname{sgn}(\sigma)} \frac{\partial}{\partial e_{\sigma(1)}} \otimes \frac{\partial}{\partial e_{\sigma(2)}} \otimes x_{\sigma(3)}
$$

or, in more invariant terms, multiplication by $x_{1} \wedge x_{2} \wedge x_{3}$. For $a=b=2, c=0$, we get $\vartheta$ from $\Theta$ if in addition we compose with the multiplication map $\mathbb{C}^{3} \otimes \mathbb{C}^{3} \rightarrow \mathrm{Sym}^{2} \mathbb{C}^{3}$ and the equivariant projection $\operatorname{Sym}^{2} \mathbb{C}^{3} \otimes\left(\mathbb{C}^{3}\right)^{\vee} \rightarrow V(2,1)$.

Hence in the basis

$$
\left\{\frac{1}{2} e_{1}^{2}, e_{1} e_{2}, e_{1} e_{3}, \frac{1}{2} e_{2}^{2}, e_{2} e_{3}, \frac{1}{2} e_{3}^{2}\right\}
$$


of $V(2,0)$ a computation with e.g. Macaulay 2 yields

$$
M=\left(\begin{array}{cccccc}
0 & \delta_{311} & -\delta_{211} & \delta_{321} & -\delta_{221}+\delta_{331} & -\delta_{231} \\
-\delta_{311} & 0 & \delta_{111}-\delta_{221}-\delta_{331} & \delta_{322} & \delta_{121}-\delta_{222}+\delta_{332} & \delta_{131}-\delta_{232} \\
\delta_{211} & -\delta_{111}+\delta_{221}+\delta_{331} & 0 & -\delta_{121}+\delta_{332} & -\delta_{131}-\delta_{232}+\delta_{333} & -\delta_{233} \\
-\delta_{321} & -\delta_{322} & \delta_{121}-\delta_{332} & 0 & \delta_{122} & \delta_{132} \\
\delta_{221}-\delta_{331} & -\delta_{121}+\delta_{222}-\delta_{332} & \delta_{131}+\delta_{232}-\delta_{333} & -\delta_{122} & 0 & \delta_{133} \\
\delta_{231} & -\delta_{131}+\delta_{232} & \delta_{233} & -\delta_{132} & -\delta_{133} & 0
\end{array}\right) .
$$

Here we abbreviated $\delta_{i j k}=\frac{\partial}{\partial x_{i}} \frac{\partial}{\partial e_{j}} \frac{\partial}{\partial e_{k}}$.

The point is that this matrix drops rank on Bateman seven tuples of points. In other words they are contained in the associated Pfaffian divisor $D \subset \mathbb{P} V(1,2)$ :

Proposition 2.6 Consider the map

$$
a: V(2,0) \otimes V(1,2) \mapsto V(0,2)
$$

induced by $m$. Then for given $Q \in V(0,2)$ and $C \in V(0,3)$, hence given Bateman seven-tuple $b\left(Q^{*}, C\right) \in V(1,2)$, there is a dual quadric $Q^{\prime} \in V(2,0)$ in the kernel of our skew matrix, i.e. with $a\left(Q^{\prime}, b\left(Q^{*}, C\right)\right)=0$. In fact, one may take $Q^{\prime}=Q^{*}$.

Proof Look at the diagram

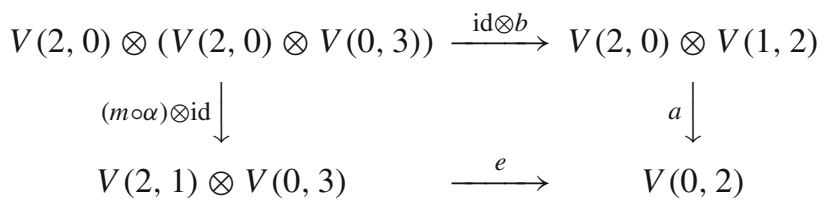

(where $\alpha: V(2,0) \otimes V(2,0) \rightarrow \Lambda^{2} V(2,0)$ is simply anti-symmetrization). One checks

- The map e exists and is nontrivial since $V(0,2)$ is contained in $V(2,1) \otimes V(0,3)$.

- The diagram commutes since there is just one non-zero map (up to a factor) from $V(2,0) \otimes(V(2,0) \otimes V(0,3))$ to $V(0,2)$ : the summand $V(0,2)$ is contained with multiplicity 1 in $V(2,0) \otimes(V(2,0) \otimes V(0,3))$. To check this it suffices to see that $V(0,2)$ is not contained in $\operatorname{Sym}^{2} V(2,0) \otimes V(0,3)$ by the Littlewood-Richardson rule.

Thus we can calculate $a\left(Q^{\prime}, b\left(Q^{*}, C\right)\right)$ in another way now:

$$
a\left(Q^{\prime}, b\left(Q^{*}, C\right)\right)=e\left((m \circ \alpha)\left(Q^{\prime}, Q^{*}\right), C\right)
$$

which is zero for $Q^{\prime}=Q^{*}$.

Corollary 2.7 Bateman seven-tuples form a 13-dimensional family in $\mathbb{P V}(1,2)$. So there is an irreducible component of the Pfaffian hypersurface $D$, say $K$, in which Bateman seven tuples are dense. 
Proof Proposition 2.6 says that the image of the pure tensors under $b: V(2,0) \otimes$ $V(0,3) \rightarrow V(1,2)$ is not all of $V(1,2)$. By upper semi-continuity of fibre dimension it thus suffices to find a pure tensor through which passes a 1-dimensional fibre (counted projectively, otherwise a two-dimensional fibre). This is easily accomplished as follows. Fix

$$
Q^{*}=e_{1}^{2}+e_{2}^{2}+e_{3}^{2}, \quad C=x_{1}^{3}+x_{2}^{3}-x_{3}^{3}-\left(x_{1}+x_{2}+x_{3}\right)^{3} .
$$

We compute the dimension of the tangent space to the fibre through the point $\left(Q^{*}, C\right)$ of the restriction of $b$ to pure tensors (at the point $\left(Q^{*}, C\right)$ ). Let $R^{*} \in V(2,0), D \in$ $V(0,3)$ be arbitrary, and with $\epsilon$ such that $\epsilon^{2}=0$ note that the equation

$$
b\left(Q^{*}, C\right)=b\left(Q^{*}+\epsilon R^{*}, C+\epsilon D\right)
$$

is equivalent to

$$
\sum_{i=1}^{3}\left(\frac{\partial}{\partial e_{i}} R^{*} \otimes \frac{\partial}{\partial x_{i}} C+\frac{\partial}{\partial e_{i}} Q^{*} \otimes \frac{\partial}{\partial x_{i}} D\right)=0 \text { in } V(1,2)
$$

To simplify, we will write $d_{R^{*}}(\cdot)=b\left(R^{*}, \cdot\right), d_{Q^{*}}(\cdot)=b\left(Q^{*}, \cdot\right)$ (differentials w.r.t. these dual quadrics) and $\operatorname{tr}=e_{1} \otimes x_{1}+e_{2} \otimes x_{2}+e_{3} \otimes x_{3}$. Note that then the previous equation is equivalent to the existence of a linear form $l \in\left(\mathbb{C}^{3}\right)^{*}$ with

$$
d_{R^{*}} C+l \cdot \operatorname{tr}=d_{Q^{*}} D
$$

and $d_{Q^{*}}(\cdot)$ may be identified with the usual differential

$$
d(\cdot)=\sum_{i} \frac{\partial}{\partial x_{i}}(\cdot) \otimes d x_{i}: \operatorname{Sym}^{k}\left(\mathbb{C}^{3}\right)^{*} \otimes \Lambda^{l}\left(\mathbb{C}^{3}\right) \rightarrow \operatorname{Sym}^{k-1}\left(\mathbb{C}^{3}\right)^{*} \otimes \Lambda^{l+1}\left(\mathbb{C}^{3}\right) .
$$

Hence our sought-for tangent space is the space of $R^{*}$ and $l$ such that

$$
d_{Q^{*}}\left(d_{R^{*}} C+l \cdot \operatorname{tr}\right)=0
$$

which a priori amounts to 8 linear equations on the 9-dimensional space of $\left(R^{*}, l\right)(8$ equations because the equality lives in the kernel of $\left.d: \Lambda^{2}\left(\mathbb{C}^{3}\right) \otimes\left(\mathbb{C}^{3}\right)^{*} \rightarrow \mathbb{C}\right)$. But one can check (e.g. with Macaulay 2) that only 7 of these are independent whence our fibre through $\left(Q^{*}, C\right)$ is 2-dimensional as desired.

Remark 2.8 It follows from the above discussion that $K$ coincides with the so-called Morley hypersurface of [12] and [13].

We are now in a position to prove

Theorem 2.9 The moduli space $\mathfrak{M}_{B}$ of Bateman seven-tuples of points in $\mathbb{P}^{2}$ is rational. 
Proof Consider the rational map

$$
\varphi: D \rightarrow \operatorname{Grass}\left(2, \operatorname{Sym}^{2}(V)\right)
$$

assigning to $P \in D$ its kernel.

A general point of $\operatorname{Grass}\left(2, \operatorname{Sym}^{2}(V)\right)$ is a pencil of (dual) quadrics in $\mathbb{P}^{2}$ with 4 distinct base points which in turn determine the pencil. Acting by $\mathrm{SL}_{3}(\mathbb{C})$ we may suppose that these points are the four vertices of a frame of reference in $\mathbb{P}^{2}$ and so $\mathrm{SL}_{3}(\mathbb{C})$ acts with a dense orbit in $\operatorname{Grass}\left(2, \operatorname{Sym}^{2}(V)\right)$. The stabilizer of a generic point in $\operatorname{Grass}\left(2, \operatorname{Sym}^{2}(V)\right)$ inside $\operatorname{PSL}_{3}(\mathbb{C})$ is the symmetric group $\mathfrak{S}_{4}$.

Claim The Morley hypersurface $K$ is the unique irreducible component of $D$ which dominates Grass(2, $\left.\operatorname{Sym}^{2}(V)\right)$.

To prove the Claim, it suffices to exhibit a Bateman seven-tuple of points such that the kernel of the skew-matrix determined by this and by $m$ consists of two general conics. One can check that for our previous choice of $Q^{*}$ and $C$ from Corollary 2.7

$$
Q^{*}=e_{1}^{2}+e_{2}^{2}+e_{3}^{2}, C=x_{1}^{3}+x_{2}^{3}-x_{3}^{3}-\left(x_{1}+x_{2}+x_{3}\right)^{3}
$$

we get a Bateman 7-tuple which when substituted into $M$ of formula (5) yields the pencil

$$
\left\langle e_{1}^{2}+e_{2}^{2}+e_{3}^{2}, e_{1}^{2}-e_{1} e_{2}+e_{2}^{2}+e_{1} e_{3}+e_{2} e_{3}\right\rangle
$$

with smooth base locus, i.e. $(1: \pm i \sqrt{2}: 1)$ and $( \pm i \sqrt{2}: 1: 1)$.

Hence $F / \mathfrak{S}_{4}$, where $F$ is the generic fibre of $\varphi$, is birational to $\mathfrak{M}_{B}$ by Corollary 2.7 and the preceding discussion. We will determine $F$ as $\mathfrak{S}_{4}$-representation. The irreducible representations of $\mathfrak{S}_{4}$ are 1 (trivial), $\epsilon$ (sign representation), $V_{2}$ (2-dimensional induced from permutation representation of $\mathfrak{S}_{3}$ ), $V_{3}$ (3-dimensional permutation representation), $V_{3}^{\prime}=V_{3} \otimes \epsilon$. Then

$$
\operatorname{Sym}^{2}(V)=V_{3}+V_{2}+1
$$

whence

$$
\Lambda^{2} V(2,0)=\Lambda^{2}\left(V_{3}+V_{2}+1\right)=\Lambda^{2} V_{2}+V_{2} \otimes\left(V_{3}+1\right)+\Lambda^{2}\left(V_{3}+1\right) .
$$

We get $F=\Lambda^{2}\left(V_{3}+1\right)=\Lambda^{2} V_{3}+V_{3} \otimes 1=V_{3}^{\prime}+V_{3} . \mathbb{P}\left(V_{3}^{\prime}+V_{3}\right) / \mathfrak{S}_{4}$ is rational since it is generically a vector bundle over $\mathbb{P}\left(V_{3}\right) / \mathfrak{S}_{4}$ which is a unirational (hence rational) surface. This completes the proof of the Theorem.

Acknowledgments Both authors were supported by the German Research Foundation [Deutsche Forschungsgemeinschaft (DFG)] through the Institutional Strategy of the University of Göttingen.

Open Access This article is distributed under the terms of the Creative Commons Attribution Noncommercial License which permits any noncommercial use, distribution, and reproduction in any medium, provided the original author(s) and source are credited. 


\section{References}

1. Bateman, H.: The quartic curve and its inscribed configurations. Am. J. Math. 36, 357-386 (1914)

2. Bogomolov, F.: Rationality of the moduli of hyperelliptic curves of arbitrary genus (Conf. Alg. Geom., Vancouver 1984). CMS Conf. Proceedings, vol. 6, pp. 17-37. Amer. Math. Soc., Providence (1986)

3. Böhning, Chr., Graf v. Bothmer, H.-Chr.: A Clebsch-Gordan formula for $\mathrm{SL}_{3}(\mathbb{C})$ and applications to rationality. Adv. Math. 224, 246-259 (2010)

4. Böhning, Chr., Graf v. Bothmer, H.-Chr.: The rationality of the moduli spaces of plane curves of sufficiently large degree. Invent. Math. 179(1). doi:10.1007/s00222-009-0214-6 (2010). preprint available at arXiv:0804.1503

5. Dolgachev, I., Kanev, V.: Polar covariants of plane cubics and quartics. Adv. Math. 98, 216-301 (1993)

6. Dolgachev, I.: Rationality of fields of invariants. In: Proceedings of Symposia in Pure Mathematics. vol. 46, pp. 3-16 (1987)

7. Dolgachev, I.: Lectures on Invariant Theory. London Mathematical Society Lecture Note Series, vol. 296, Cambridge University Press, Cambridge (2003)

8. Grace, J.H., Young, W.H.: The Algebra of Invariants. Cambridge University Press, Cambridge (1903); reprinted by Chelsea Publ. Co., New York (1965)

9. Katsylo, P.I.: Rationality of orbit spaces of irreducible representations of SL 2 . Izv. Akad. Nauk SSSR, Ser. Mat. 47(1), 26-36 (1983); English Transl.: Math USSR Izv. 22, 23-32 (1984)

10. Katsylo, P.I.: Rationality of the moduli spaces of hyperelliptic curves. Izv. Akad. Nauk SSSR Ser. Mat. 48, 705-710 (1984)

11. Katsylo, P.I.: On the birational geometry of $\left(\mathbb{P}^{n}\right)^{(m)} / \mathrm{GL}_{n+1}$, Max-Planck Institut Preprint, MPI/94144 (1994)

12. Morley, F.: On the Lüroth quartic curve. Am. J. Math. 41, 279-282 (1919)

13. Ottaviani, G., Sernesi, E.: On the hypersurface of Lüroth quartics, to appear in Michigan Math. J. preprint 2009, arXiv:0903.5149 